\title{
Managing insulin resistance: role of liraglutide
}

This article was published in the following Dove Press journal:

Clinical Pharmacology:Advances and Applications

23 June 2010

Number of times this article has been viewed

\author{
Sanjay Kalra' \\ Bharti Kalra' \\ Satish Kumar ${ }^{2}$ \\ Amit Sharma' \\ 'Department of Endocrinology, Bharti \\ Hospital, Karnal, India; ${ }^{2}$ Department \\ of Clinical Operations, Excel Life \\ Sciences, Noida, India
}

\begin{abstract}
Diabetes mellitus is part of the insulin resistance syndrome, which includes hypertension, dyslipidemia, and obesity as its other components. Conversely, insulin resistance is a major pathophysiologic factor in the development of type 2 diabetes. It makes sense, therefore, to choose an anti-diabetic medication that acts on insulin resistance and its clinical components, while having anti-hyperglycemic effects as well. This review discusses the non-glycemic, or extra-pancreatic effects, including insulin sensitization, of liraglutide, a novel GLP-1 analog.
\end{abstract}

Keywords: liraglutide, GLP-1 analog, weight modulation, systolic blood pressure, cardioprotection, insulin resistance

\section{Introduction}

Diabetes mellitus is part of the insulin resistance syndrome (IRS), which includes hypertension, dyslipidemia, and obesity as its other components. In the initial stages insulin resistance is compensated by increased insulin secretion. However the disease is progressive, with continuous $\beta$-cell failure leading to inadequately increased insulin secretion in relation to the degree of insulin resistance. Present data show that in insulin resistance, the incretin hormone (GIP and GLP-1) responses to a mixed meal are impaired and are related to the degree of insulin resistance. ${ }^{1}$ In this regard therapeutic use of GLP-1 analogs offers a novel approach to address insulin resistance.

Liraglutide is a GLP-1 analog with $97 \%$ homology to the amino acid sequence of human GLP-1. ${ }^{2}$ The attachment of $\mathrm{C} 16$ fatty acid chain helps delay absorption of liraglutide by forming a heptamer, and delays degradation by DPP-4. ${ }^{3}$ Liraglutide is thus able to effectively mimic the actions of mature GLP-1, and improve insulin secretion as well as response.

\section{Glycemic effects}

Liraglutide reduces plasma glucose levels in both fasting and post prandial state, thus achieving improvement in hemoglobin $\mathrm{A}_{1 \mathrm{c}}\left(\mathrm{HbA}_{1 \mathrm{c}}\right){ }^{4}$ It does so by increasing insulin secretion, reducing post-prandial glucagon secretion, delaying gastric emptying, improving $\beta$-cell function, and reducing appetite. ${ }^{5}$

The glucoregulatory effects, and results of clinical trials with respect to glycemic control, are discussed in detail in other reviews. ${ }^{5}$ 


\section{Extra-glycemic/pleiotropic effects}

Liraglutide has been shown to have beneficial effects on other components of the IRS, such as body weight, blood pressure and lipids. This article reviews the recent data from animal and clinical studies.

\section{Energy intake}

In animal studies, liraglutide has been shown to be associated with a reduction in dietary calorie intake and a change in food preference. In one study, female rats made obese by candy-feeding were randomized to $0.2 \mathrm{mg} / \mathrm{kg}$ liraglutide twice daily, $10 \mathrm{mg} / \mathrm{kg}$ vildagliptin twice daily, vehicle only or chow-only diet. The rats were subjected to oral glucose tolerance tests, energy expenditure measurement, and body composition analysis by dual-energy $\mathrm{x}$-ray absorptiometry, as well as histology to assess pancreatic- $\beta$-cell mass. Control animals developed weight gain as well as fat gain.

Liraglutide administration fully reversed the weight and fat gains noted with candy-feeding, decreased calorie intake, and shifted food preference from candy to chow. Liraglutide did not reduce energy expenditure in spite of weight loss, thus highlighting another dimension of its mechanism of action. ${ }^{6}$

Liraglutide may have the ability to change food preferences, and thus reduce energy intake in human beings as well, as evidenced by anecdotal reports. Similar results have been seen in clinical studies. Liraglutide given as a $1.8 \mathrm{mg}$ daily dose reduced hunger in two studies. A similar dose was noted to reduce energy intake in one of these studies. However, a lower dose $(0.6 \mathrm{mg} \%)$ was not associated with such effects. ${ }^{7-9}$

\section{Body weight}

Liraglutide $1.2 \mathrm{mg}$ and $1.8 \mathrm{mg}$ have been found to reduce body weight in most studies. In the LEAD-3 trial, which compared subcutaneous liraglutide once daily with oral glimepiride $8 \mathrm{mg}$ once daily for 52 weeks in 746 subjects of type 2 diabetes, body weight fell by $2 \mathrm{~kg}$ and $2.5 \mathrm{~kg}$ respectively in the $1.2 \mathrm{mg}$ and $1.8 \mathrm{mg}$ liraglutide group, but increased by $1.1 \mathrm{~kg}$ in the glimepiride group $(P<0.0001$ for both liraglutide groups vs glimepiride). ${ }^{10}$

The weight loss was maximal during first 16 weeks of therapy, and was not related to nausea. Weight remained stable over one year, after the initial improvement.

Another study was done over 24 weeks to compare a lower dose of liraglutide ( $0.9 \mathrm{mg}$ once daily) $(\mathrm{n}=268)$ and glibenclamide $(\mathrm{n}=132)$ in Japanese patients. It found a weight loss of $1.91 \mathrm{~kg}$ in the liraglutide group as compared to glibenclamide group $(P<0.0001)$ in spite of the lower dose used, and better glycemic control. ${ }^{11}$

Similar results have been noted in studies using liraglutide as combination therapy. In studies LEAD-2 $(\mathrm{n}=1087)$, LEAD-4 $(\mathrm{n}=533)$ and LEAD-5 $(\mathrm{n}=581)$, liraglutide led to significant reductions in weight, across the full spectrum of baseline body mass index. ${ }^{12-14}$ Maximal weight loss was noted in patients with a higher baseline BMI $\left(\geq 35 \mathrm{~kg} \mathrm{~m}^{-2}\right) .{ }^{15}$

In the LEAD-1 study, which compared addition of various doses of liraglutide with rosiglitazone and placebo to baseline glimepiride therapy, weight change seen with liraglutide $1.2 \mathrm{mg}$ and $1.8 \mathrm{mg}$ was significantly different from rosiglitazone (1.8 kg difference for the $1.2 \mathrm{mg}$ group and $2.3 \mathrm{~kg}$ for the $1.8 \mathrm{mg}$ group). However, the difference was not significant when compared with the placebo group. ${ }^{16}$

In the LEAD-2 trial, which compared addition of liraglutide to glimepiride or placebo on a base of metformin, liraglutide $0.6 \mathrm{mg}$ showed a $2.8 \mathrm{~kg}$ difference, liraglutide $1.2 \mathrm{mg}$ had a $3.6 \mathrm{~kg}$ difference and liraglutide $1.8 \mathrm{mg}$ had $3.8 \mathrm{~kg}$ difference with respect to glimepiride. All these differences were significant $(P \leq 0.01) .^{12}$

The LEAD-4 trial compared liraglutide $1.2 \mathrm{mg}$ and $1.8 \mathrm{mg}$ with placebo, when added to a metformin and rosiglitazone combination. The difference in weight with respect to placebo, was $1.6 \mathrm{~kg}$ and $2.6 \mathrm{~kg}$ for the $1.2 \mathrm{mg}$ and $1.8 \mathrm{mg}$ liraglutide groups $(P<0.0001){ }^{13}$

A similar level of statistical significance was noted for liraglutide $1.8 \mathrm{mg}$, versus insulin (difference in weight change $3.43 \mathrm{~kg}$ ) in the LEAD-5 trial, where the baseline therapy was metformin and glimepiride. ${ }^{14}$

Weight gain or excessive body weight is also associated with public distress. The LEAD-2 reported this domain in the Impact of Weight on Quality of Life questionnaire, and found less distress due to body weight with liraglutide than with glimepiride $(P<0.02){ }^{17}$

The weight loss that occurs with liraglutide is predominantly of visceral fat. ${ }^{18}$ Available preclinical and clinical data clearly demonstrate the utility of liraglutide in reducing weight, thus making it a drug of choice for obese patients with diabetes. Weight loss has been demonstrated to improve therapeutic outcomes and reduce complications in overweight diabetics. The correction of this important part of insulin resistance syndrome, should, in principle, lead to improved cardiovascular outcomes. This should in turn encourage the use of liraglutide as a drug of choice for diabetes.

Studies are also planned to assess the effect of liraglutide in obese, but non-diabetic individuals. 


\section{Blood pressure}

Hypertension is another component of insulin resistance, and frequent comorbid condition of diabetes, which need to be addressed.

Systolic blood pressure reduced by $3.6 \mathrm{mmHg}$ with liraglutide $1.8 \mathrm{mg}(P<0.0118$ versus glimepiride), and by $2.1 \mathrm{mmHg}$ with liraglutide $1.2 \mathrm{mg}$ ( $P=$ NS vs glimepiride) in the LEAD-3 trial. Non-significant decreases in diastolic blood pressure were reported in the same study. ${ }^{19}$

These results have led to the realization that liraglutide is effective not only in correcting hyperglycemia but also has pleiotropic effects such as blood pressure lowering. Similar results have been reported from other LEAD trials, where liraglutide has been studied in combination with other drugs.

Liraglutide $1.8 \mathrm{mg}$ has demonstrated lowering of systolic blood pressure by 1.4-4.5 mmHg in LEAD-1, -2 and -5 , reaching statistical significance in the latter $(P<0.05)$. The reduction noted with liraglutide was significant when compared with the reduction of systolic blood pressure seen with placebo in LEAD-4. ${ }^{12-14}$ No significant change in diastolic blood pressure has been noted.

\section{Insulin resistance}

Measures of insulin resistance, ie, homeostasis model assessment of insulin resistance (HOMA-IR), have been studied in LEAD trials.

In LEAD-3, the monotherapy trial, insulin resistance decreased by $0.65 \%$ with liraglutide $1.2 \mathrm{mg}$ ( $P=0.0249$ vs glimepiride) and $1.35 \%$ with liraglutide $1.8 \mathrm{mg}(P=0.0011$ vs glimepiride), while increasing by $0.85 \%$ in the glimepiride group. No significant differences were reported between groups of indices of $\beta$-cell function. ${ }^{20}$

LEAD-1 and -2, however, did not report any improvement in insulin sensitivity with liraglutide. ${ }^{20}$

\section{Cardiovascular markers}

Liraglutide has been shown to reduce triglyceride levels, without affecting concentration of other lipids.$^{21}$ This makes it an attractive choice for patients with diabetic dyslipidemia, which is characterized by high levels of triglycerides.

While no long term data of cardiovascular outcomes is available, other studies show a cardio protective effect of liraglutide. The drug reduces levels of B-type natriuretic peptide and plasminogen activator inhibitor-1 (PAI-1) in humans. ${ }^{22}$ Similar effects on PAI-1 and ICAM-1 have been demonstrated in vitro. Healthy volunteers given liraglutide have shown no effect on cardiac repolarization (corrected QT interval). ${ }^{23}$

\section{Tolerability}

Liraglutide is generally well tolerated. The most common adverse events are gastrointestinal, ie, nausea, vomiting, and diarrhea, and decrease over time. Hypoglycemia occurs rarely, and no major hypoglycemic events have been reported with liraglutide monotherapy (LEAD-3), liraglutide + metformin (LEAD-2), and liraglutide + metformin + rosiglitazone (LEAD-4). ${ }^{20}$

In LEAD-3, the incidence of minor hypoglycemia was 0.30 and 0.25 events/patient/year with liraglutide $1.2 \mathrm{mg}$ and $1.8 \mathrm{mg} /$ day, as compared to 1.96 events with glimepiride (both $P<0.0001$ ). After 2 years, the rates were 0.16 and 0.28 for the liraglutide group, and 1.82 events/patient/year for glimepiride $(P<0.0001$ and $P<0.0001$ respectively).

As liraglutide appears to be safe, well-tolerated, and associated with a negligible incidence of hypoglycemia, it may find a place in the management of insulin resistance syndrome.

\section{Conclusion}

Liraglutide is a GLP-1 analogue with proven efficacy, safety, and tolerability as a glucose lowering drug, when used in type 2 diabetes mellitus. A detailed analysis of its pleiotropic or extra glycemic effects, however, reveals a multifaceted character of the molecule. Liraglutide is effective in reducing body weight, decreasing visceral fat, lowering systolic blood pressure, and improving lipid profile as well as other cardiovascular risks markers, while reducing insulin resistance.

These beneficial effects on all components of the insulin resistance syndrome, or metabolic syndrome, with minimal risk of hypoglycemia, make liraglutide an attractive option for management of insulin resistance.

\section{Disclosures}

The authors report no conflict of interest.

\section{References}

1. Rask E, Olsson T, Söderberg S, et al. Impaired incretin response after a mixed meal is associated with insulin resistance in nondiabetic men. Diabetes Care. 2001;24(9):1640-1645.

2. Irie S, Matsumura Y, Zdravonovic M, et al. Tolerability, pharmacokinetics and pfarnacodynamics of the once-daily human GLP-1 analogue liraglutide in Japanese subjects: a randomized, double blind, placebo- controlled dose escalation study. Int J Clin Pharmacol Ther. 2008;46(6):273-279.

3. Steensgaard DB, Thomsen JK, Olsen HB, Knudsen LB. The molecular basis for the delayed absorption of the once daily human GLP-1 analogue: liraglutide [abstract nr 552-P]. Diabetes. 2008;57 Suppl 1:A164.

4. Juhl CB, Hollingdal M, Sturis J, et al. Bedtime administration of NN2211, a long acting GLP-1 derivative, substantially reduces fasting and post parandial glycemia in type 2 diabetes. Diabetes. 2002;51(2): 424-429. 
5. Kalra S, Kalra B, Sharma A. Liraglutide- a novel GLP-1 analogue. Recent patents Endocr Metabol Immune Drug Discovery. 2009;3:200-204.

6. Raun K, Von Voss P, Gotfredsen CF, Golozoubova V, Rolin B, Knudsen LB. Liraglutide, a long-acting glucagon-like peptide-1 analogue, reduces body weight and food intake in obese candy-fed rats, whereas a dipeptidyl peptidase-IV inhibitor, vildagliptin, does not. Diabetes. 2007;56:8-15.

7. Harder H, Nielson H, Tu DT, Astrup A. The effect of liraglutide, a long acting glucagons like peptide-1 derivative, on glycemic control, body composition and 24-h energy expenditure in patients with type 2 diabetes. Diabetes Care. 2004;27(8):1915-1921.

8. Horowitz M, Flint A, Doran S, et al. Effect of the once daily human GLP-1 analogue liraglutide on appetite and energy intake in type 2 diabetes [abstract nr 889]. Diabetologia. 2008;51 Suppl 1:S355.

9. Flint K, Kapitza C, Hindsberger C, Zdravkovic M. The once-daily human GLLP-1 analogue liraglutide decreases postprandial hunger and energy intake [abstract nr 555-P]. Diabetes. 2008;57 Suppl 1:A165.

10. Vilsboll T, Zdravkovik M, LeThi T, et al. Liraglutide, a long acting human glucagons-like peptide-1 analog, given as monotherapy significantly improves glycaemic control and lowers body weight without risk of hypoglycemia in patients with type 2 diabetes. Diabetes Care. 2007;30:1608-1610.

11. Seino Y, Rasmussen MF, Katayama Y, et al. Liraglutide is statistically superior to glibenclamide at controlling glicemia and weight, with hypoglycemic risk, and improves beta-cell functions in Japanese subjects with T2D [abstract nr 536-P]. 69th Scientific sessions of the American Diabetes Association; 2009 Jun; New Orleans (LA).

12. Nauck M, Frid A, Harmensen K, et al. Efficacy and safety comparison of liraglutide, glimepiride, and placebo, all in combination with metformin in type 2 diabetes; the LEAD (liraglutide effect and action in diabetes)-2 study. Diabetes Care. 2009;32(1):84-90.

13. Zinman B, Gerich J, Buse JB, et al. Efficacy and safety of the human glucagons like peptide-1 analogue liraglutide in combination with metformin and thiazolidinedione in patients with type 2 diabetes (LEAD-4 Met + TZD). Diabetes Care. 2009;32(7):1224-1230.

14. Russell-Jones D, Vaag A, Schimitz O, et al. Liraglutide vs insulin glargine and placebo in combination with metformin and sulphonylureas therapy in type 2 diabetes mellitus (LEAD- 5 met + SU): a randomizes controlled trial. Liraglutide effects and action in diabetes-5 (LEAD-5) met + SU study group. Diabetologia. Epub 2009 Aug 14 .
15. Schimitz O, Russell-Jones D, Shaw J, et al. Liraglutide, a human GLP-1 analogue, reduces body weight in subjects with type 2 diabetes, irrespective of body mass index at baseline [abstract nr 888]. Diabetologia. 2008;51 Suppl 1:S354.

16. Marre M, Shaw J, Brabdle M, et al. Liraglutide, a once-daily human GLP-1 analogue added to a sulphonylurea over 26 weeks produces greater improvement in glycemic and weight control compared with adding rosiglitazone or placebo in subjects with type 2 diabetes (LEAD-1 SU). Diabet Med. 2009;26(3):268-278.

17. Frid A, Nauck MA, Harmensen K, et al. Evaluation of patient reported outcomes in subjects with type 2 diabetes treated with the once-daily human GLP-1, analogue liraglutide or glimepiride, both as add on to metformin [abstract nr 2080-PO]. Diabetes. 2008;57 Suppl 1:A574-A575.

18. Jendle J, Nauck MA, Mathews D, et al. The reduction of body weight with liraglutide, 1 once-daily human GLP-1 analogue in type 2 diabetes, primarily comes from fat tissue and the fat tissue lost in predominantly visceral fat [abstract nr 797]. Diabetologia. 2008;51 Suppl 1:S318.

19. Colagiuri S, Frid A, Zdravkovic M, et al. Liraglutide, a human GLP-1 analogue reduces systolic blood pressure in subjects with type 2 diabetes [abstract nr 899]. Diabetologia. 2008;51 Suppl 1:S360.

20. Garber A, Henry R, Ratner R, et al. Liraglutide versus glimepiride monotherapy for type 2 diabetes (LEAD-3 Mono): a randomized, 52 week, phase 3, double-blind, parallel treatment trial. Lancet. 2009;373(9662):473-481.

21. Courreges J-P, Vilsboll T, Zdravkovic M, et al. Beneficial effects once-daily liraglutide, a human glucagons like peptide-1 analogue, on cardiovascular risk biomarkers in patients with type 2 diabetes. Diabet Med. 2008;25(9):1129-1131.

22. Dear AE, Liu H, Knudsen LB, et al. Liraglutide inhibits hyperglycemia induced expression of PAI-1 and vascular adhesion molecules in an in vitro model of endothelial cell dysfunction [abstract nr 893]. Diabetologia. 2008;51 Suppl 1:S356.

23. Sprenger C, Khutoryansky N, Chaterjee DJ, Zdravkovic M, Litwin J. Liraglutide does not affect cardiac repolarization [abstract nr 2103]. Diabetes. 2008;57 Suppl 1:581.
Clinical Pharmacology: Advances and Applications

\section{Publish your work in this journal}

Clinical Pharmacology: Advances and Applications is an international, peer-reviewed, open access journal publishing original research, reports, reviews and commentaries on all areas of drug experience in humans. The manuscript management system is completely online and includes a very quick and fair peer-review system, which is all easy to use.

\section{Dovepress}

Visit http://www.dovepress.com/testimonials.php to read real quotes from published authors. 